\title{
Smart Phone Application for Connected Vehicles and Smart Transportation
}

\author{
Yingqi Yue \\ University of Toronto \\ danielyyq@cs.toronto.edu
}

\author{
Kaiwen Zhang \\ University of Toronto \\ kzhang@cs.toronto.edu
}

\author{
Hans-Arno Jacobsen \\ University of Toronto \\ Jacobsen@eecg.utoronto.ca
}

\begin{abstract}
Real-time traffic analysis is an interesting research topic across different disciplines and areas. In order to provide a better and practical way of representing and gathering traffic data, we need to have a suitable media with high mobility and qualitative performance such as smartphones. This paper proposes a smartphone application which presents and collects traffic information data to improve the travelling experience. The application is based on a server-client model, developed in Java and deployed on the Android platform.
\end{abstract}

\section{Categories and Subject Descriptors}

C.2.4 [Distributed System]: Distributed applications;

H.3.4 [System and Software]: Distributed systems

\section{General Terms}

Algorithms, Design, Reliability

\section{Keywords}

Smartphone application, traffic prediction, location awareness, crowd sourcing

\section{INTRODUCTION}

Highway traffic analysis today is a Big Data problem which involves gathering real time event streams from loop detectors and VDS (Vehicle Detecting System). Message-oriented middleware such as publish/subscribe [1] can be used in this scenario to support crowd-sourced sensing applications of traffic data.

This paper introduces a smartphone-based application for traffic analysis which performs three major types of functionalities: (i) Simple, user-friendly mobile interface to display real-time highway traffic condition. (ii) Give certain predictions for seasonal traffic conditions based on historical highway data. (iii) Monitor users' GPS positions and keep individual traces for future analysis [2].

Each of these features will be further discussed in the below sections.

\section{DATA SOURCES ANALYSIS}

ONE-ITS ${ }^{1}$ provides highway traffic data around Toronto in three major types: (i) On-road traffic cameras images captured in real-time, (ii) Event streams which contains the speed, volume and occupancy of highway loop detectors or VDS. (iii) Lists of all incidents reported within a certain time period.

The sensor data provided by ONE-ITS is imperfect due mainly to hardware issues. A typical incomplete sensor stream includes missing events or windows of events with null or static value for certain attributes (e.g., the lane speed or occupancy). These missing or incorrect data can lead to mistakes if we take them into account for further traffic analysis, so a reliable source data error detection system needs to be built on top of the existing dissemination layer (eg. publish/subscribe) to protect against errors. We built a file string parser which scans through the entire sample traffic data files and import them into a database. We profile the sample stream to detect which blocks of information are misleading. We then integrate the parameters found on the sample to detect errors in the online feeds. Error reconciliation is performed by assigning a new average value using extrapolated data from nearby sensors in the same time frame.

We also employ crowdsourced location-based traffic data as a secondary source of data to estimate traffic conditions where the sensor data is missing or incomplete. Different weights are assigned to each user trace based on how much confidence the uploaded information is. As more people give similar traffic conditions on city streets, more information will be gathered and displayed in order of accuracy.

\section{TRAFFIC PREDICTION}

The purpose of traffic prediction is to compare seasonal traffic differences and display it to the user for itinerary duration estimation. In order to perform the prediction, users need to specify the start and destination points. The algorithm run the server side will process the historical traffic data and return not only the current estimated time of arrival but also different seasonal estimation time (eg. the same itinerary in the winter).
Permission to make digital or hard copies of all or part of this work for personal or classroom use is granted without fee provided that copies are not made or distributed for profit or commercial advantage and that copies bear this notice and the full citation on the first page. To copy otherwise, or republish, to post on servers or to redistribute to lists, requires prior specific permission and/or a fee.

Middleware 2013 Posters and Demos Track, December 9-13, 2013, Beijing, China Copyright 2013 ACM 978-1-4503-2549-3 ...\$15.00.
${ }^{1}$ ONE-ITS gets real-time traffic data from Ministry of Transportation Ontario, City of Toronto; The project pages are located at: http://one-its-webapp1.transport.utoronto.ca 


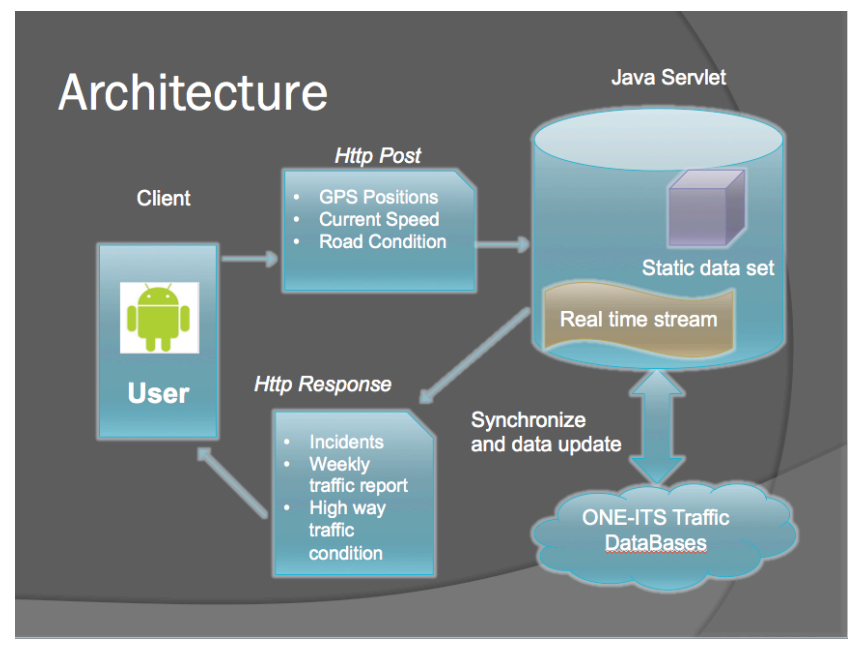

Figure 1: Internal architecture of the smartphone project

By grouping multiple continuous sensor records, we can get the Highway average speed and traffic flow volume at certain query time between two boundary points by averaging instantaneous speed over distance.

Another critical point is to calculate the real road distance for the itinerary. Our approach is to separate the itinerary into small pieces by referencing each traffic sensor's GPS position. Due to the fact that sensors are relatively close to each other on highways, we can approximate the distance using straight-line segments, which we then combine to get the total road distance. The Haversine Fomula [3] below can be used to calculate great-circle distances or shortest distances between two points on Earth mathematically:

$$
\begin{aligned}
& \Delta \text { lat }=\mid \text { lat2- lat } 1 \mid \\
& \Delta \text { long }=\mid \text { long2- long } 1 \mid \\
& a=\sin ^{2}\left(\frac{\Delta \text { lat }}{2}\right)+\cos (\text { lat } 1) \times \cos (\text { lat } 2) \times \sin ^{2}\left(\frac{\Delta \text { long }}{2}\right) \\
& c=2 \times \arctan (\sqrt{\mathrm{a}} / \sqrt{1-\mathrm{a}}) \\
& \mathrm{d}=\mathrm{R} \times \mathrm{c}
\end{aligned}
$$$$
\mathrm{d}=\text { distance in kilometers }
$$

By using traffic prediction as described above, we can generate reports on traffic patterns with different time granularities. On a daily basis, the highway traffic will reach its highest load during morning and evening rush hours. In a congestion situation, traffic standing waves, the center of the congestion, and the moving speed of the center can be detected. Since a vehicle accelerates when the space gap $g$ to the preceding vehicle is greater than a synchronization space gap $G$ and decelerates when $g$ is smaller than the safe space gap $g_{\text {save }}$ due to Kerner's three-phase traffic theory, our traffic prediction system can notify drivers of highway congestion ahead of time to prevent acceleration into traffic jams. On a monthly and seasonal time window, traffic loads vary among different highways: for instance, the highway average speed during the winter is the slowest due to ice and snow conditions.

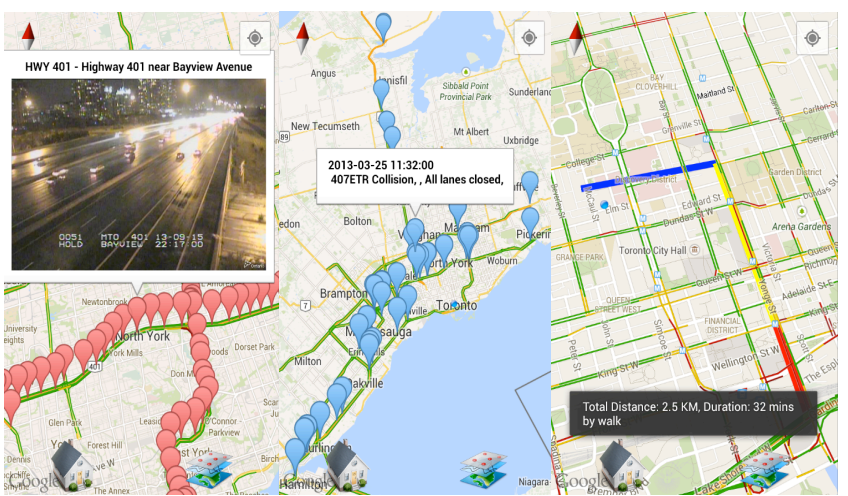

Figure 2: Real-time Traffic Camera layer (left), Highway incidents layer (middle) and user historical trace view (right) in android client demo

\section{ARCHITECTURE AND LIVE DEMO}

Figure 1 depicts the internal architecture of the smartphone project. The notable components are the Android client that sends and receives traffic information, the Java servlet on the server side storing user locations as well as getting feeds from the ONEITS service, and the database that maintains and captures all highway traffic data.

The client uses the Google Play location service to record user GPS positions and calculate the speed of the user to determine the type of movement (e.g., by car, bicycle or foot). User information is sent to our server periodically. Data packages are stored on the user phone in SQLite during offline periods.

On the server side, a static data set contains historical traffic data from the ONE-ITS database for traffic prediction. The server also collects a real-time stream of incident notifications, camera images and highway sensor data. Traffic prediction calculation is offloaded from the client to the server.

In our live demonstration, the audience will see different layers of the traffic information system as well as a video on the generation of a sample user historical color-coded trace as shown in Figure 2. There will be an introduction and demonstration of the traffic prediction functionality. We will also demonstrate the crowdsourcing functionalities of our application using user location-based traffic data and show how the accuracy of the data changes according to the volume of users.

\section{REFERENCES}

[1] Fidler, E., Jacobsen, H. A., Li, G., \& Mankovski, S. (2005, December). The PADRES Distributed Publish/Subscribe System. In FIW (pp. 12-30).

[2] Tamer Abdulazim ,Hossam Abdelgawad, Khandker M. Nurul Habib and Baher Abdulhai, "Using Smartphones and Sensor Technologies to Automate the Collection of Travel Data" 91st 43 Annual Meeting of Transportation 44 Research Board.

[3] Pornpen Ratsameethammawong and M.L.Kulthon Kasemsan "Mobile Phone Location Tracking by the Combination of GPS, Wi-Fi and Cell Location Technology" Volume 2010 